\title{
Relationship of Heart Rate Variability (HRV) Parameters Including pNNxx With the Subjective Experience of Stress, Depression, Well-Being, and Every-Day Trait Moods (TRIM-T): A Pilot Study
}

\author{
Michael Trimmel
}

Institute of Environmental Hygiene, Centre of Public Health, Medical University of Vienna, Vienna, Austria

\begin{abstract}
Heart rate variability (HRV) is an established indication of cardiovascular regulation and is often not only considered in relation to health, but also to subjective aspects of perceived affect. However, as the various HRV parameters are not identical but interrelated, they vary in regard to different aspects of every-day moods, stress, and depression. Therefore it is suggested that those HRV parameters would discriminate perceived affect. This study compares the relationship of HRV parameters to aspects of every-day affect. In 60 persons heart rate (HR) and HRV measures of time domain parameters (including $\mathrm{pNNxx}$ parameters), frequency domain parameters, and relative parameters (from twenty-four hour electrocardiography recordings) were investigated in relation to perceived stress (PSQ), anxiety (STAI-T), depression (BDI-II), general well-being (GWBS), and traits of every-day moods (TRIM-T). Results showed that HRV parameters were correlated to most subjective measures (PSQ, BDI-II, GWBS, and good mood and relaxation in TRIM-T). Low pNNxx parameters (pNN10 - pNN40) correlated negatively with stress and depression and positively with well-being, whereas high pNNxx parameters (pNN125 - pNN175), SDNN, ultralow frequency (ULF), and total power (TP) were in particular correlated to good mood. Furthermore, results showed that slow rhythms, in particular ULF, were correlated to positive affect (good mood, relaxation), but not to negative affect (stress, anxiety, depression). Correlations of cardiovascular activity with well-being are less pronounced, indicating that the construct of well being may be seen as a mixture of positive affect and low stress - and is in general less related to cardiovascular activity than stress and/or good mood. For relative HRV measures, in particular for $\log \mathrm{LF} / \mathrm{HF}$ (which is often interpreted as a measure of autonomic balance), no correlations with every-day affect were observed. Results further indicate that the time domain parameters of pNNxx (in particular pNN10 - pNN40 vs. pNN125 - pNN175) are differentially related to negative/positive affect, contrary to heart rate, and may therefore be considered as an objective indication of affect.
\end{abstract}

Keywords: Affect, autonomic balance, cardiac physiology, cardiovascular regulation, emotion, health.

\section{INTRODUCTION}

Heart rate variability (HRV) denotes the variation of timespan from one heart beat to the next one, i.e. from R- to $\mathrm{R}$-wave in the electrocardiogram (ECG) of "normal to normal beats" (therefore sometimes called NN beats). Based on a huge amount of evidence [1] the variations of the interplay of the sympathetic and parasympathetic activity of the autonomous nervous system (ANS), which are tightly linked to inhibitory pathways in the brain [2-4], are supposed to be underlying mechanisms of HRV. For the quantification of HRV, besides geometrical methods, the analysis by the decomposition in frequency bands (by fast Fourier transformation or autocorrelative algorithms) of NN intervals resulting in bands labeled as high frequency (HF; 0.15-0.40 $\mathrm{Hz}$ ), low frequency (LF; 0.04-0.15 Hz), very low frequency (VLF; 0.0033-0.04 Hz), ultra-low frequency (ULF; 0-0.0033 $\mathrm{Hz}$ ), and total power (TP) as well as measures in the time domain based on the NN- (but at least the RR-) time interval

*Address correspondence to this author at the Medical University of Vienna, Centre of Public Health, Institute of Environmental Hygiene, Kinderspitalgasse 15, A-1090 Vienna, Austria; Tel: +4314016034911; Fax: +43140160934903; E-mail: michael.trimmel@meduniwien.ac.at
(SDNN, RMSSD, pNN50) is common. Thus, there are various measures of HRV, some of them suggested to be related (e.g. HF with pNN50 [1, 5]), but not to be identical. However, there is only scarce converging evidence on the sensitivity of the parameters to physiological mechanisms and psychological constructs, except $\mathrm{HF}$, for which converging evidence for vagal activation exists.

Depending on the respective model in mind and the preassumptions of the researcher, specific parameters are commonly selected. For example, when viewing HRV as a general phenomenon of flexibility of the autonomic nervous system, SDNN and TP were found to be useful, especially with the supposed relationship with aspects of health $[1,6$, 7].

In particular the model of distinguishing parasympathetic from sympathetic activity led to using HF power as an index of parasympathetic activity and LF power as an index of sympathetic activation [1]. However this approach is challenged by the fact that LF is associated with sympathetic activation as well as with parasympathetic activation. Therefore also the suggested measure of LF/HF as an index of "autonomous balance" is deteriorated [8, 9]. By measuring sympathetic activation with impedance cardiography, Berntson et al. [10] suggested a model in which the interplay 
of parasympathetic and sympathetic activations is not necessarily antagonistic but could also take place simultaneously in the sense of co-activation or co-deactivation.

A recent approach suggested to broaden the traditional pNN-analysis by not only taking into account the percentage of consecutive NN-intervals differing more than $50 \mathrm{~ms}$ (pNN50), but also taking into account other limits of the NN-intervals (pNNxx). That was proven to be fruitful in relation to health (differentiating patients with congestive heart failure from healthy persons [11]), neuroautonomic dysfunction of psychiatric disorders [12], obstructive sleep apnoea [13], depression [14], and physiological load induced by a fast increase in altitude by ascent with cable car [15].

The justification to analyze pNNxx is based on the observation that pNN50 shows a floor effect (see upper part of figure 2 in Mietus et al. [11]), especially in cases of high HR and coincidental low HRV. The measures in the range from 5 - $30 \mathrm{~ms}$ (i.e. pNN05 - pNN30) did not show that effect and could therefore be more sensitive than the traditional pNN50 to figure out aspects in physiological response. Another reason to analyze $\mathrm{pNNxx}$ is the expectation that various $\mathrm{NN}$-intervals of up to $200 \mathrm{~ms}$ would reflect different qualities of HRV.

There are numerous works on the relationship of HRV with health or health-related aspects like physical fitness or effects of stress and life events. However, there are only very few investigations on subjective experience of affect in relation to $\mathrm{HRV}$, and these are most often limited to a single construct (e.g. depression [14]). In this study the differing relationship of various HRV measures to stress, anxiety, depression, well-being, and every-day moods was investigated. It was expected that different HRV parameters, in particular those of the pNNxx family, reflect different aspects of subjective experience of affects in every-day life.

\section{METHOD}

\subsection{Subjects}

Sixty-six persons of European origin participated in the study unpaid and voluntarily. However, due to recording problems, HR from only 60 persons (aged $18-52, \mathrm{M}=26.8$, $\mathrm{SD}=6.24 ; 18$ females) could be analyzed. Test persons had a body mass index from 18.4 to $29.0(\mathrm{M}=22.83$, SD = $2.64)$, only healthy persons were included. The test persons consisted of 23 university students, 25 employees, 7 selfemployed persons, 4 high-school students, and 1 person seeking work. Twenty-three of the test persons had a university degree, 31 test persons had an academic high school diploma with at least 12 years of schooling, and 5 persons had had 9 years of schooling as their highest level of education. Twenty-seven of the test persons were single, 33 had a steady relationship. Thirty-four of the test persons reported to have carried out physical training on the days of the 24-hour recordings.

\subsection{Design}

The study followed a one shot design by correlating HR and traditional HRV parameters (SDNN, RMSSD, pNN50, ULF, VLF, LF, HF, TP, log LF/HF, ULF\%, VLF\%, LF\%,
$\mathrm{HF} \%$ ) as well as pNNxx-parameters (pNN05 - pNN200) from 24-hour ECG recordings with subjective experience by ratings on perceived stress (PSQ), anxiety (STAI-T), depression (BDI-II), well-being (GWBS) and trait moods (TRIM-T).

\subsection{Self-Assessment Instruments}

PSQ. The revised Perceived Stress Questionnaire [16] assesses perceived stress during the past two weeks in adults with 20 items on a four-point Likert type scale ranging from 1 (almost never) to 4 (usually). It measures four aspects of stress (worries, tension, demands, and [-]joy), resulting in a general perceived stress dimension. A Cronbach's alpha of 0.92 for healthy adults was reported for this instrument [16].

STAI-T. The Trait Anxiety Inventory [17] assesses trait anxiety with 20 items each on a four-point Likert type scale ranging from 1 (almost never) to 4 (almost always). For this instrument an average internal consistency of Cronbach's alpha of 0.89 was reported [18].

BDI-II. The revised Beck Depression Inventory [19] assesses the intensity of depression during the past two weeks with 21 items. Each of the items consists of four statements about a symptom of depression, the statements being arranged in increasing severity. For this questionnaire a Cronbach's alpha of 0.89 was reported for a non-clinical sample [20].

GWBS. The General Well-Being Schedule [21] assesses general well-being during the past month with 14 items rated on a labeled 6-point Likert scale type ranging from 0 (most negative option) to 5 (most positive option) and 4 items on an 11-point scale with the endpoints 0 (most negative option) to 10 (most positive option). A Cronbach's alpha of 0.91 was found for this schedule [22].

TRIM-T. Trimmel's Index of Trait Moods is based on the circumplex model of affect [23] with the axes degree-ofarousal and pleasure (see Fig. 1). The author developed a 16item German questionnaire, the TRIM-T (Appendix A). Subjects are asked to rate every-day moods on a 5-point Likert scale ranging from 1 to 5 with the labels "never (nie) rarely (selten) - sometimes (gelegentlich) - often (oft) always (immer)" following the introductory sentence "In general, I am ... (Im Allgemeinen bin ich ...)". A prior factor analysis of data from a large data set suggested four dimensions. First, "energy" (Energie) with the items fresh (frisch), energized (energiegeladen), [-]tired (müde), []sleepy (schläfrig), second "good mood" (Gute Stimmung) with the items good mood (guter Stimmung), cheerful (heiter), [-]depressed (deprimiert), [-]downhearted (niedergeschlagen), third "motivation" (Motivation) with the items willing to show effort (anstrengungsbereit), willing to perform (leistungsmotiviert), [-]bored (gelangweilt), []indifferent (gleichgültig), and fourth "relaxation" (Entspannung) with the items relaxed (entspannt), calm (gelassen), [-]nervous (nervös), [-]restless (unruhig). Cronbach's alpha in the present study revealed acceptable to good consistencies: "energy" $\alpha=0.73$, "good mood" $\alpha=$ 0.75 , "motivation" $\alpha=0.68$, "relaxation" $\alpha=0.79$ ). Whereas the dimensions energy (from energized to tired/sleepy) and good mood (from good mood to bad mood) are directly related to Russell [23], the dimensions motivation (from 
motivated to bored) and relaxation (from relaxed to anxious) differ from Russell's model [23], as the moods investigated in the TRIM-T seem to be more realistic and essential in assessing every-day moods in private and in business contexts.

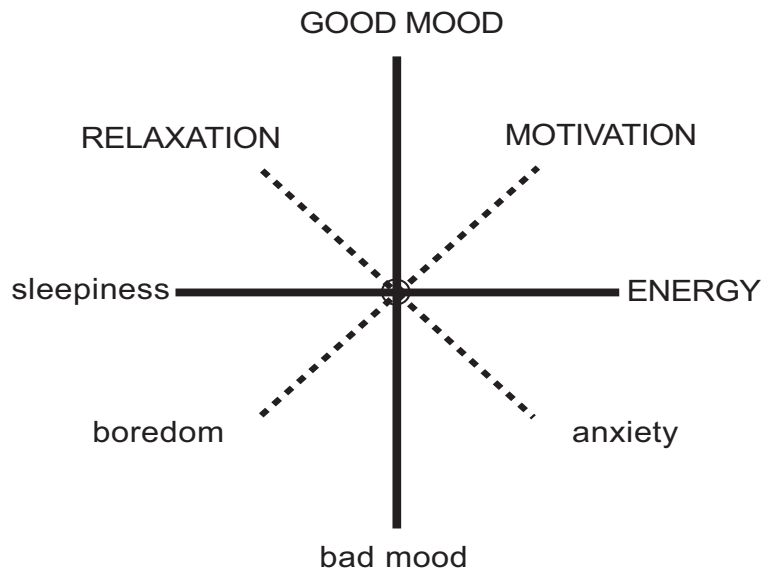

Fig. (1). Structure and dimensions of Trimmel's index of moods.

\subsection{Physiological Recording and Analysis}

Electrocardiography was gained by a modified Lead II configuration and analyzed by the program Medilog Darwin (http://schiller.at). After visual inspection of the signal, 24hour HR and HRV parameters (time-domain, frequency domain, and relative scores as $\log \mathrm{LF} / \mathrm{HF}$ and percentages of the total power in the frequency domain as $\mathrm{HF} \%$ etc.) from $\mathrm{NN}$-intervals (at least 5 consecutive valid and recognized as normal PQRST waves) were computed. For the parameters of the pNNxx-family, besides intervals of $>50 \mathrm{~ms}$ (pNN50) also intervals of greater than $5,10,20,25,30,40,60,70,75$, $80,90,100,125,150,175$, and $200 \mathrm{~ms}$ were considered for the measures pNN05 - pNN200.

\subsection{Procedure}

German speaking test persons were fitted with ambulatory ECG recording devices (Medilog AR12 Holter ECG recorders from Schiller-Engineering, Graz, Austria, http://schiller.at) in the afternoon to take recordings of at least 24 hours. Then they filled in the questionnaires (German versions) and were asked to come back on the next day to return the devices.

\subsection{Statistical Analysis}

Statistical analysis and the testing of the respective assumptions were performed with the program IBM SPSS Statistics 22 .

\section{RESULTS}

Descriptive statistics (mean and $S D$ ) of investigated variables as well as correlation coefficients of $\mathrm{HR}$ and $\mathrm{HRV}$ parameters with subjective experiences of stress, anxiety, depression, well-being, and every-day moods are presented in Table 1. Patterns of correlations with a $95 \%$ CI not including 0 (as computed by IBM SPSS22 bootstrapping) were compared for the relationship of physiological parameters with the questionnaires.

Results showed correlations with a $95 \%$ CI not including 0 of HRV parameters with perceived stress (PSQ), depression (BDI-II), general well-being (GWBS) as well as with good mood and relaxation (TRIM-T).

With the exceptions of motivation and energy, correlations with subjective ratings were found in the pNNxx family, with highest correlations of pNN10 - pNN40 to perceived stress and to depression. GWBS displayed relatively high correlations with pNN20 - pNN50, whereas good mood was predominantly (highly) correlated with pNN100 - pNN175 and relaxation with pNN125 - pNN175.

Other time domain parameters related to subjective ratings were RMSSD (negatively correlated to PSQ and positively to GWBS) and SDNN (positively correlated to good mood and relaxation).

Correlations with a $95 \%$ CI not including 0 of HRV measures from frequency domain showed positive correlations to good mood in all frequency bands (ULF, VLF, LF, HF, TP) and to relaxation in ULF, VLF, and TP. With the exception of HF the powers of all other frequency bands were negatively correlated to PSQ.

For relative HRV measures no significant correlations with a $95 \%$ CI not including 0 with subjective measures were observed; in particular not for $\log \mathrm{LF} / \mathrm{HF}$, for which only a marginal trend $(r=0.21, p=0.105$, two-tailed $)$ with energy was observed.

In HR, correlations with a $95 \% \mathrm{CI}$ not including 0 were positive with PSQ, STAI-T, BDI-II and negative with GWBS, good mood, and relaxation. The correlations of HRV measures with the questionnaire data showed opposite correlations.

\section{DISCUSSION}

Ratings of subjective qualities of affect and stress were found to be correlated to HRV in general as expressed by time domain parameters, in particular that from the pNNxx family. Parameters from frequency domain were in particular highly correlated to good mood and to relaxation, but also to stress, depression, and well-being.

For relaxation correlations appeared to be very systematic in the frequency domain. Ultra- and very low frequencies (ULF, VLF, and TP) are positively correlated to relaxation whereas in higher frequencies (LF and HF) 0 is within the CI. Frequency domain parameters (except HF), were negatively correlated with stress. For good mood all parameters of the frequency domain were relatively highly and positively correlated. Thus, frequency domain measures are positively correlated to positive affect, negatively to stress, and - at least in this study - not correlated to trait anxiety (STAI-T), depression, and well-being. Results also indicate that frequency domain parameters (from ULF up to HF) differ only gradually in the correlations with subjective measures; in particular the differences between LF and HF are marginal. Furthermore the negative correlation of LF with stress challenges the model that LF reflects sympathetic 
Table 1. Descriptive statistics ( $M$ and $S D)$ of variables and correlation coefficients $(r)$ of $H R$ and HRV parameters with subjective measures of perceived stress (PSQ), trait anxiety (STAI-T), Beck's depression inventory (BDI-II), general well-being (GWBS), and trait moods (TRIM-T). Correlations with a 95\% CI not including 0 are displayed in italics and highest coefficients (not less than 0.01 below the highest ones) in each block of a column are displayed in bolds.

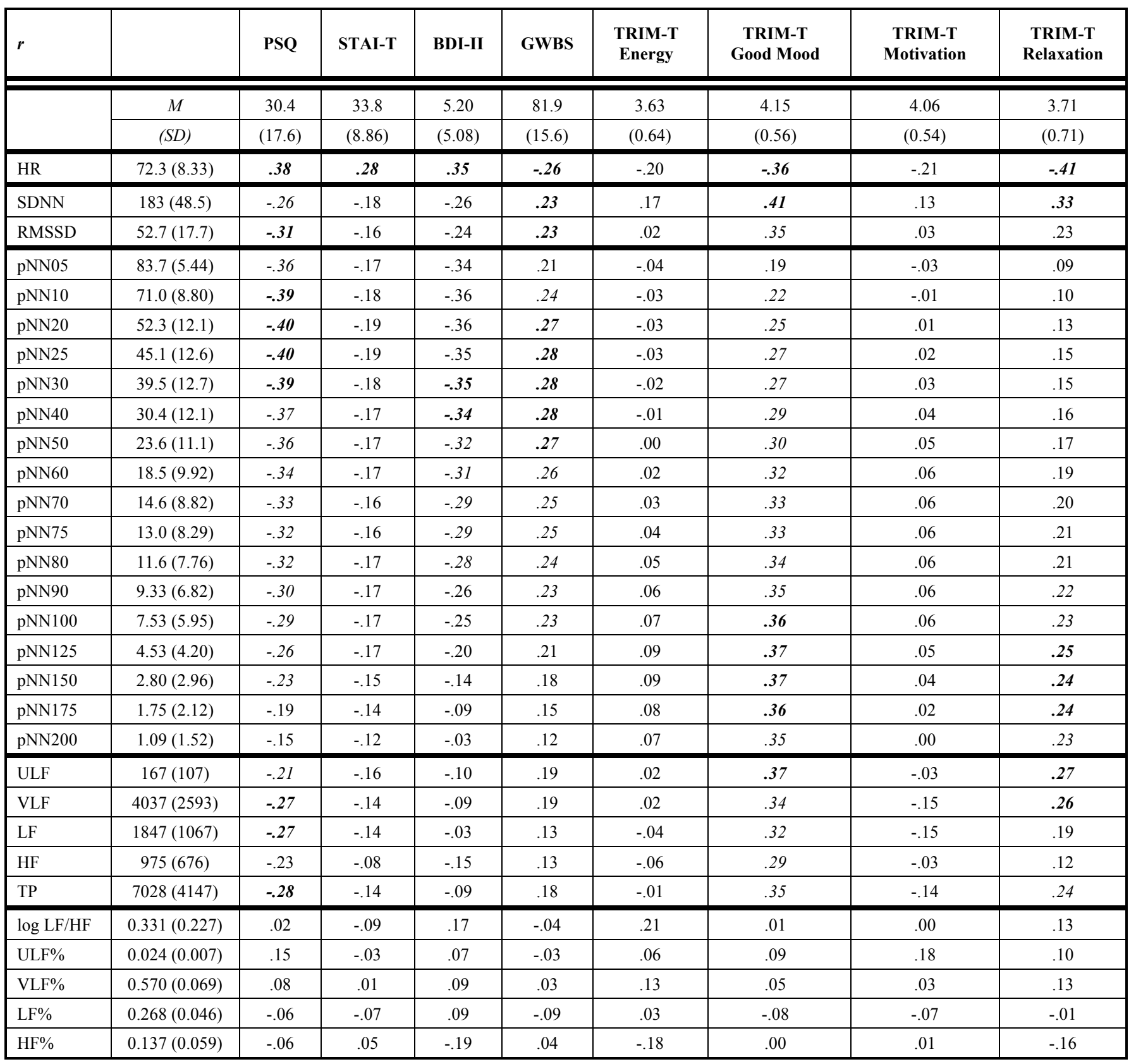

activation, as in that case one would expect a positive correlation.

Contrary to frequency domain parameters, measures of the pNNxx family showed a more specific pattern. Whereas low pNNxx parameters, especially pNN20 - pNN40, are highly negatively correlated to stress and depression and to some extent positively to well-being, high pNNxx parameters ( $\mathrm{pNN} 100$ - $\mathrm{pNN} 175)$ are highly correlated to good mood and relaxation. Thus results indicate that low pNNxx parameters are more related to negative affect whereas high $\mathrm{pNNxx}$ parameters are more related to positive affect.
Only for well-being this distinction does not appear in a vivid form. Interestingly, general well-being was not as highly correlated to HRV as good mood, perceived stress, and depression. This can be seen in line with a recent suggestion [24] that the relation of HRV to subjective wellbeing is "mediated by the habitual use of strategies of emotion regulation that involve executive functions", with the well-known impact of positive affect on health in general [25], and even with social cognition [26]. An alternative interpretation could be that the construct of well-being may be seen as a mixture of experiencing positive affect (good mood) together with the absence of stress, thus resulting in a measure reflecting both aspects. 
High periodicity of HRV in general as indicated by TP as well as by all other frequency bands (ULF, VLF, LF, HF) and in time domain parameters of SDNN and RMSSD as well as in pNNxx parameters were in particular correlated to good mood. A somewhat comparable effect was observed in an early work, but using short-term recordings for affects of appreciation [27]. Contrary to positive affect, negative affect was inversely correlated to HRV in general.

HR too was correlated to subjective measures, with the exception of energy and motivation, for which no relationship was found in the present study. It is speculated that these dimensions are more correlated to short-term measures at particular physiological states than to 24 hour recordings. HR was in particular highly negatively correlated with relaxation, good mood, and well-being and positively correlated with stress, anxiety and depression. Such a pattern of correlations indicates that $\mathrm{HR}$ is not able to distinguish between positive and negative affect, contrary to HRV parameters, in particular parameters of pNNxx. Moreover, to differentiate affect by HRV, measures of high pNNxx parameters combined with ULF could furthermore be considered as an index of good mood.

Empirical evidence on the impact of trait anxiety on 24hour HRV seems to be rare, whereas for short term measures and in particular for state anxiety a reduced HRV has been observed [28, 29]. In the present study only $\mathrm{HR}$ was correlated to trait anxiety, but no measure of HRV. This is in line with the conclusion of Dishman et al. [30] that vagal modulation of HR is sensitive to perceived emotional stress, regardless of a person's disposition towards experiencing anxiety.

Relative HRV parameters did not show any significant relationship to ratings on subjective qualities of affect, in particular no correlation (not including 0) of $\log \mathrm{LF} / \mathrm{HF}$ was observed.

Furthermore, the viewpoint that $\mathrm{HF}$ and pNN50 are highly correlated $[1,5]$ was not supported by the present study. Whereas pNN50 was found to be negatively correlated to stress and depression, and positively with wellbeing and good mood, HF was only correlated to good mood.

Limitations of the study can be seen in the relatively small sample of rather young, well educated, healthy, and rather athletic persons with an unequal distribution of sex. A further weakness can be seen in the fact that the amount of physical activity within the recording period was not included in the analysis. As in this study only 24-hour ECG recordings were correlated to "general" aspects of affect in the sense of the trait-concept, the relationship of cardiovascular characteristics to state-related emotions remains unclear.

Altogether, it was observed that LF and HF activity are only gradually differing in the relationship to subjective measures. This also challenges the model that LF/HF would be an index of the traditional concept of autonomic balance. LF and HF showed also less discrimination in the relationship to affect than ULF and VLF. Furthermore, results indicate that negative affect like stress and depression are negatively related to HRV with highest correlations found in pNN10 - pNN40. Positive affect was highly correlated to a general high HRV and particularly expressed by ULF as well as by time domain measures of SDNN and of pNNxx around pNN125. One can suggest that the differentiating aspects of pNNxx can be considered as an objective indication of assessing affect in every-day moods in private and business contexts.

\section{APPENDIX A}

\section{IN GENERAL, I am ...}

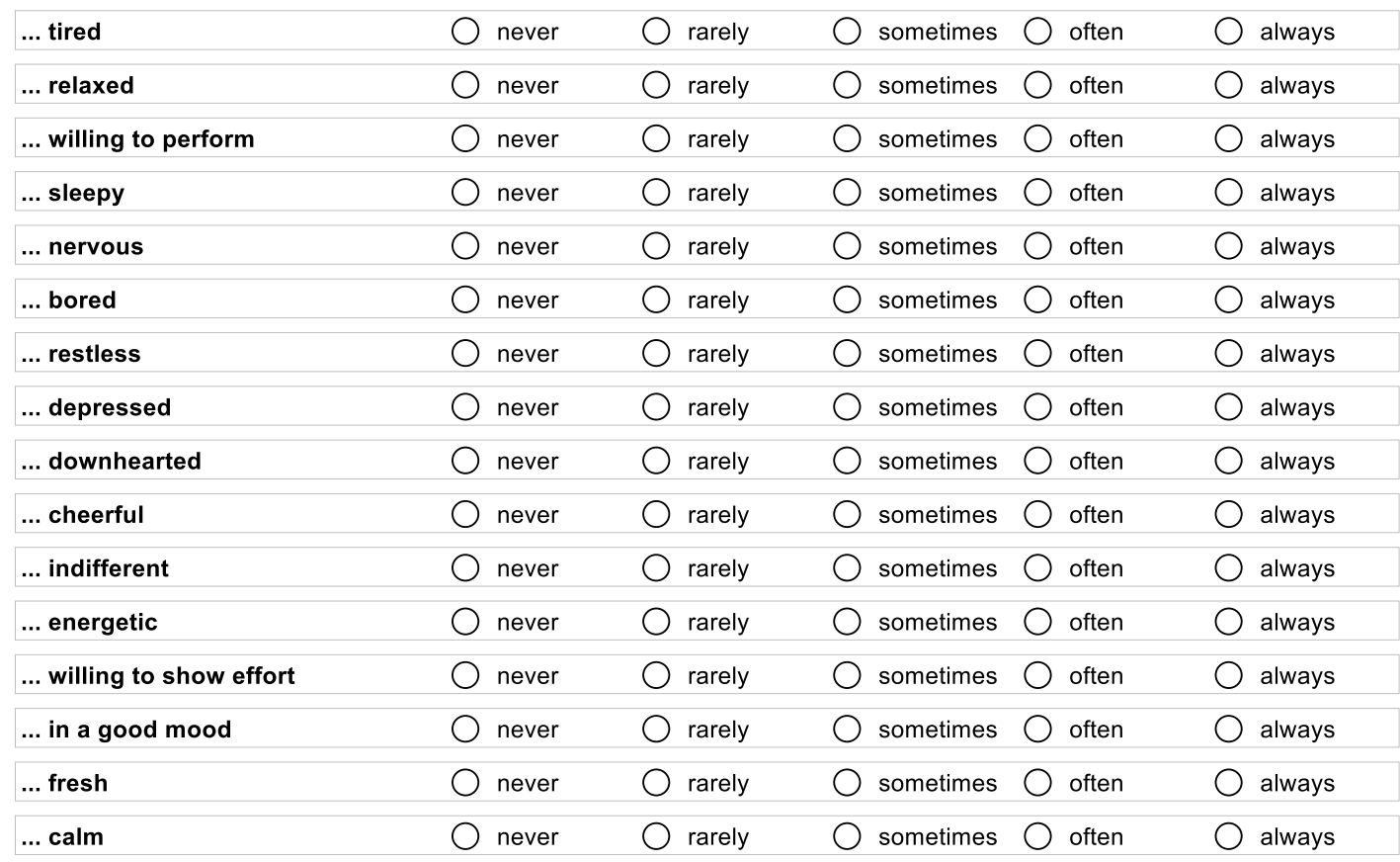




\section{CONFLICT OF INTEREST}

The author confirms that this article content has no conflict of interest.

\section{ACKNOWLEDGEMENTS}

The author wants to thank O. Höppe for his help in data collection and $\mathrm{G}$. Langer for the help in preparing the manuscript.

\section{REFERENCES}

[1] Task Force of the European Society of Cardiology (ESC) and the North American Society of Pacing Electrophysiology (NASPE). Heart rate variability: standards of measurement, physiological interpretation and clinical use. Europ Heart J 1996; 17: 354-81.

[2] De Morree HM, Szabò BM, Rutten G-J, Kop, WJ. Central nervous system involvement in the autonomic responses to psychological distress. Neth Heart J 2013; $21: 64-9$.

[3] Samuels MA. The brain-heart connection. Circulation 2007; 116: 77-85.

[4] Thayer JF, Lane RD. Claude Bernard and the heart-brain connection: Further elaboration of a model of neurovisceral integration. Neurosci Biobehav R 2009; 33: 81-8.

[5] Goedhart AD, van der Sluis S, Houtveen JH, Willemsen G, de Geus EJ. Comparison of time and frequency domain measures of RSA in ambulatory recordings. Psychophysiology 2007; 44: 20415 .

[6] Billman GE. Heart rate variability - a historical perspective. Front Physiol 2011; 2: 86 .

[7] Kemp AH, Quintana DS. The relationship between mental and physical health: insights from the study of heart rate variability. Int J Psychophysiol 2013; 89(3): 288-96.

[8] Eckberg, L. Sympathovagal balance: a critical appraisal. Circulation 1997; 96: 3224-32.

[9] Billman GE. The LF/HF ratio does not accurately measure cardiac sympatho-vagal balance. Front Physiol 2013; 4: 26.

[10] Berntson GG, Bigger JT, Eckberg DL, et al. Heart rate variability: origins, methods and interpretative caveats. Psychophysiology 1997; 34: 623-48.

[11] Mietus JE, Peng CK, Henry I, Goldsmith RL, Goldberger AL. The pNNx files: re-examining a widely used heart rate variability measure. Heart 2002; 88: 378-80.

[12] Kim J, Yi SH, Ahn YM, et al. The pNNx heart rate variability statistics: an application to neuroautonomic dysfunction of clozapine-treated subjects. Psychiatr Investig 2009; 6(4): 296-8.

[13] Balachandran JS, Rahangdale S, Yim-Yeh S, et al. PNN10 and pNN20, markers of vagal tone, are decreased in obese patients with obstructive sleep apnea compared to controls. Am J Respir Crit Med 2011; 183: A2212.
[14] Leistedt SJ-J, Linkowski P, Lanquart J-P, et al. Decreased neuroautonomic complexity in men during an acute major depression episode: analysis of heart rate dynamics. Transl Psychiatry 2011; 1: e27.

[15] Trimmel K. Sensitivity of HRV parameters including pNNxx proven by short-term exposure to $2700 \mathrm{~m}$ altitude. Physiol Meas 2011; 32(3): 275-85.

[16] Fliege H, Rose M, Arck P, et al. The perceived stress questionnaire (PSQ) reconsidered: validation and reference values from different clinical and healthy adult samples. Psychosom Med 2005; 67: 7888.

[17] Spielberger C, Gorsuch R, Lushene, R. State-trait anxiety inventory (STAI). Palo Alto, CA: Consulting Psychology Press 1970.

[18] Barnes L, Harp D, Jung WS. Reliability generalization of scores on the Spielberger State-Trait Anxiety Inventory. Educ Psychol Meas 2002; 63(4): 603-18

[19] Hautzinger M, Keller F, Kühner C. BDI-II; Beck depressionsinventar revision. Frankfurt am Main: Harcourt Test Services 2006.

[20] Kühner C, Bürger C, Keller F, Hautzinger M. Reliabilität und Validität des revidierten Beck-Depressionsinventars (BDI-II). Nervenarzt 2007; 78: 651-6.

[21] Dupuy HJ. The general well-being schedule. In: Mcdowell I, Newell C, Eds. Measuring health: a guide to rating scales and questionnaire. $2^{\text {nd }}$ ed. USA: Oxford University Press 1977; pp. 20613.

[22] Fazio AF. A concurrent validational study of the NCHS's genera well-being schedule. US Government Printing Office: Washington DC 1977.

[23] Russell JA. A circumplex model of affect. J Pers Social Psychol 1980; 39: 1161-78.

[24] Geisler FCM, Vennewald N, Kubiak T, Weber H. The impact of heart rate variability on subjective well-being is mediated by emotion regulation. Pers Indiv Differ 2010; 49: 723-8.

[25] Dockray S, Steptoe A. Positive affect and psychobiological processes. Neurosci Biobehav R 2010; 35: 69-75.

[26] Quintana DS, Guastella AJ, Outhred T, Hickie IB, Kemp AH. Heart rate variability is associated with emotion recognition: direct evidence for a relationship between autonomic nervous system and social cognition. Int J Psychophysiol 2012; 86: 168-72.

[27] McCraty R, Atkinson M, Tiller W, Rein G, Watkins AD. The effects of emotion on short-term power spectrum analysis of heart rate variability. Am J Cardiol 1995; 76(14): 1089-93.

[28] Miu AC, Heilman RM, Miclea M. Reduced heart rate variability and vagal tone in anxiety: trait versus state, and the effects of autogenic training. Auton Neurosci-Basic 2009:145: 99-103.

[29] Wilhelm FH, Pfaltz M.C, Grossman P, Roth WT. Distinguishing emotional from physical activation in ambulatory psychophysiological monitoring. Biomed Sci Instrum 2006; 42: 458-63.

[30] Dishman RK, Nakamura Y, Garcia ME, Thompson RW, Dunn AL, Blair SN. Heart rate variability, trait anxiety, and perceived stress among physically fit men and women. Int J Psychophysiol 2000; 37: 121-33. 